\title{
Efficient Numerical Algorithm for the Solution of Eight Order Boundary Value Problems by Haar Wavelet Method
}

\author{
Rohul Amin ${ }^{1} \cdot$ Kamal Shah ${ }^{2}$. Qasem M. Al-Mdallal ${ }^{3}$ (D) Imran Khan ${ }^{1}$. \\ Muhammad Asif ${ }^{1}$
}

Accepted: 6 February 2021 / Published online: 22 February 2021

(c) The Author(s), under exclusive licence to Springer Nature India Private Limited part of Springer Nature 2021

\begin{abstract}
In this paper, the Haar technique is applied to both nonlinear and linear eight-order boundary value problems. The eight-order derivative in the boundary value problem is approximated using Haar functions in this technique and the integration process is used to obtain the expression of the lower order derivative and the approximate solution of the unknown function. For the verification of validation and convergence of the proposed technique, three linear and two nonlinear examples are taken from the literature. The results are also compared with other methods available in the literature. Maximum absolute and root mean square errors at various collocation and Gauss points are contrasted with the exact solution. The convergence rate is also measured, which is almost equivalent to 2 , using different numbers of collocation points.
\end{abstract}

Keywords Boundary value problems - Gauss elimination method - Collocation method . Haar wavelet

Qasem M. Al-Mdallal

q.almdallal@uaeu.ac.ae

Rohul Amin

raminmath@uop.edu.pk

Kamal Shah

kamalshah408@gmail.com

Imran Khan

lec.imran@uop.edu.pk

Muhammad Asif

asif.tangi@uop.edu.pk

1 Department of Mathematics, University of Peshawar, Peshawar 25120, Khyber Pakhtunkhwa, Pakistan

2 Department of Mathematics, University of Malakand, Dir(L) 18000, Khyber Pakhtunkhwa, Pakistan

3 Deparment of Mathematical Sciences, United Arab Emirates University, P.O Box 15551, Al Ain, Abu Dhabi, United Arab Emirates 


\section{Introduction}

Boundary value problems (BVPs) with higher order arise in the field of astrophysics, hydrodynamic and hydro magnetic stability, fluid dynamics, astronomy, beam and long wave theory, applied physics and engineering. Due to their mathematical significance and their applications in different fields of applied sciences, many researchers studied higher order BVPs. These equations model the physics of hydrodynamic stability problems. When we heated from below, an infinite horizontal layer of fluid an instability is take place in side, subjected to action of rotation. The phenomena of set in the aforesaid instability as over stability is modeled by an eighth-order BVP [1]. The torsional vibration phenomena of beams were modelled as eight-order BVP by Bishop et al. [2]. Due to, the wide spread applications of eight order BVPs many researcher taken more interest in the numerical solution of these equations.

For the numerical solution, Siddiqi and Akram [3,4] implemented Nonic spline and nonpolynomial spline methods to order eight special linear BVPs models. Siddiqi has also shown in $[3,4]$ that the convergence of the above techniques is second order. By utilizing the Adomian decomposition technique Wazwaz [5] solve some special eight BVPs numerically. Siddiqi and Twizell have recently introduced a numerical technique based on polynomial degree six splines in [6] for the solution for some special types of BVPs of eight order. It was found, however, that the method diverged at the points adjacent to the boundary. Twizell et al. noted the same problem in $[10,11]$ by numerically solving some higher order BVPs. It was investigated that due to the use of lower order test functions in the aforementioned technique, the results vary. Differential Quadrature Methods (DQM) were implemented by the authors in [6], which uses high-order test functions in the domain. But the failure of the DQM solution is that it dealt with only second order BVPs. The $\delta$-point approach can be used for BVPs of higher order [10]. For descriptions of DQM technique enhancements and implementations, we refer to $[6,10]$. The improvement of the DQM leads to the generalization of (DQM) $[12,13]$. The DQM generalized is used for the solution of fourth and sixth order BVPs [13-15]. In addition, it is used in fluid mechanics for solution of linear Onsager and nonlinear Blasius problems with [12] in the third and sixth orders. The initial value problems of the second, third, and fourth orders are also solved using the [13] process. Liu et al. expanded the generalised DQM to [16] for the solution of eight-order BVPs. [17], study the computational and theoretical modeling of the transmission dynamics of novel COVID-19 under Mittag-Leffler power law. Khan et al. [18], study stability and simulation of a fractional order plant nectar pollinator model.

In this paper the Haar technique will be developed for solution of eight order BVPs. The nonlinear BVP of eight order is:

$$
u^{(8)}(t)=F\left(t, u, u^{(1)}, u^{(2)}, u^{(3)}, u^{(4)}, u^{(5)}, u^{(6)}, u^{(7)}\right), \quad a \leq t \leq b,
$$

where $F$ is given function, while in the linear BVP of eight order is:

$$
u^{(8)}(t)+f(t) u^{(4)}(t)+g(t) u(t)=h(t), \quad t \in[a, b],
$$

with the following BCs:

$$
\begin{array}{ll}
u(0)=\alpha_{1}, & u(1)=\beta_{1}, \\
u^{(1)}(0)=\alpha_{2}, & u^{(1)}(1)=\beta_{2}, \\
u^{(2)}(0)=\alpha_{3}, & u^{(2)}(1)=\beta_{3}, \\
u^{(3)}(0)=\alpha_{4}, & u^{(3)}(1)=\beta_{4},
\end{array}
$$


where $f(t), g(t)$ and $h(t)$ are given functions. In this work, we implement the haar wavelet method for the solution of eight order boundary value problems. It is worthy mentioning that the Haar wavelet method has been used recently to solve different classes of integral and differential equations; see for example [7-9].

The paper is written as: in "Haar Wavelet" section Haar functions are defined. Numerical HWC technique for solution of both nonlinear and linear eight-order BVPs are given in "Numerical Scheme for Eight Order BVP" section. In "Numerical Examples" section, some problems are given for validation purpose. Conclusion is given at the last "Conclusion" section.

\section{Haar Wavelet}

Haar function on interval $\left[\gamma_{1}, \gamma_{2}\right)$ is given by [19]

$$
h_{1}(t)=\left\{\begin{array}{lc}
1, & \text { for } t \in\left[\gamma_{1}, \gamma_{2}\right), \\
0, & \text { otherwise }
\end{array}\right.
$$

The mother wavelet on $\left[\gamma_{1}, \gamma_{2}\right)$ is

$$
h_{2}(t)= \begin{cases}1, & \text { for } t \in\left[\gamma_{1}, \frac{\gamma_{1}+\gamma_{2}}{2}\right) \\ -1, & \text { for } t \in\left[\frac{\gamma_{1}+\gamma_{2}}{2}, \gamma_{2}\right) \\ 0, & \text { otherwise }\end{cases}
$$

All the other terms in the HW series can be represented in $t \in\left[\rho_{1}, \rho_{2}\right)$ except the scaling function

$$
h_{i}(t)= \begin{cases}1, & \text { for } t \in\left[\rho_{1}, \rho_{2}\right), \\ -1, & \text { for } t \in\left[\rho_{2}, \rho_{3}\right), \\ 0, & \text { otherwise }\end{cases}
$$

where

$$
\begin{aligned}
& \rho_{1}=\gamma_{1}+\left(\gamma_{2}-\gamma_{1}\right) \frac{\zeta}{d}, \\
& \rho_{2}=\gamma_{1}+\left(\gamma_{2}-\gamma_{1}\right) \frac{\zeta+0.5}{d}, \\
& \rho_{3}=\gamma_{1}+\left(\gamma_{2}-\gamma_{1}\right) \frac{\zeta+1}{d},
\end{aligned}
$$

where integer $d=2^{r}$, where $r=0,1, \ldots, r^{\prime}$ and let the integer $\zeta=0,1, \ldots, d-1$. In $[0,1], \rho_{1}, \rho_{2}$ and $\rho_{3}$ are defined as:

$$
\rho_{1}=\frac{\zeta}{d}, \quad \rho_{2}=\frac{\zeta+0.5}{d}, \quad \rho_{3}=\frac{\zeta+1}{d} .
$$

Any member of $L^{2}[0,1)$, is expressed as:

$$
u(t)=\sum_{k=1}^{\infty} \lambda_{k} h_{k}(t) .
$$


For approximation purposes, this series is truncated at finite $N$ terms

$$
u(t) \approx \sum_{k=1}^{N} \lambda_{k} h_{k}(t) .
$$

We use the symbol

$$
p_{i, 1}(t)=\int_{0}^{t} h_{i}(t) d x
$$

and

$$
p_{i, 1}(t)= \begin{cases}t-\rho_{1}, & \text { for } t \in\left[\rho_{1}, \rho_{2}\right) \\ \rho_{3}-t, & \text { for } t \in\left[\rho_{2}, \rho_{3}\right) \\ 0, & \text { elsewhere }\end{cases}
$$

Thus value of $p_{i, 2}$ is

$$
\begin{aligned}
& p_{i, 2}(t)=\int_{0}^{t} p_{i, 1}(s) d s, \\
& p_{i, 2}(t)= \begin{cases}\frac{1}{2}\left(t-\rho_{1}\right)^{2}, & \text { if } t \in\left[\rho_{1}, \rho_{2}\right), \\
\frac{1}{4 m^{2}}-\frac{1}{2}\left(\rho_{3}-t\right)^{2}, & \text { if } t \in\left[\rho_{2}, \rho_{3}\right), \\
\frac{1}{4 m^{2}}, & \text { if } t \in\left[\rho_{3}, 1\right), \\
0, \text { elsewhere. } & \end{cases}
\end{aligned}
$$

Also the value of $p_{i, 3}$ is given by

$$
p_{i, 3}(t)=\int_{0}^{t} p_{i, 2}(s) d s,
$$

we obtain

$$
p_{i, 3}(t)= \begin{cases}\frac{1}{6}\left(t-\rho_{1}\right)^{3}, & \text { if } t \in\left[\rho_{1}, \rho_{2}\right) \\ \frac{1}{4 m^{2}}\left(t-\rho_{2}\right)-\frac{1}{6},\left(\rho_{3}-t\right)^{3} & \text { if } t \in\left[\rho_{2}, \rho_{3}\right) \\ \frac{1}{4 m^{2}}\left(t-\rho_{2}\right), & \text { if } t \in\left[\rho_{3}, 1\right) \\ 0, & \text { elsewhere }\end{cases}
$$

Similarly, the value of $p_{i, 4}$ is given by

$$
p_{i, 4}(t)=\int_{0}^{t} p_{i, 3}(s) d s,
$$

by simplifying this integral we obtain

$$
p_{i, 4}(t)= \begin{cases}\frac{1}{24}\left(t-\rho_{1}\right)^{4}, & \text { if } t \in\left[\rho_{1}, \rho_{2}\right), \\ \frac{1}{8 m^{2}}\left(t-\rho_{2}\right)^{2}-\frac{1}{24},\left(\rho_{3}-t\right)^{4}+\frac{1}{192 m^{4}}\left(\rho_{3}-t\right)^{3} & \text { if } t \in\left[\rho_{2}, \rho_{3}\right), \\ \frac{1}{8 m^{2}}\left(t-\rho_{2}\right)^{2}+\frac{1}{192 m^{4}}, & \text { if } t \in\left[\rho_{3}, 1\right), \\ 0, & \text { elsewhere. }\end{cases}
$$

Generally,

$$
p_{i, n}(t)=\int_{0}^{t} p_{i, n-1}(t) d x .
$$


Thus $p_{i, n}(t)$ is obtained as under [19],

$$
p_{i, n}(t)= \begin{cases}0, & \text { for } t \in\left[0, \rho_{1}\right), \\ \frac{\left(t-\rho_{1}\right)^{n}}{n !}, & \text { for } t \in\left[\rho_{1}, \rho_{2}\right), \\ \frac{\left[\left(t-\rho_{1}\right)^{n}-2\left(\rho_{1}-\rho_{2}\right)^{n}\right]}{n !}, & \text { for } t \in\left[\rho_{2}, \rho_{3}\right), \\ \frac{1}{n !}\left[\left(t-\rho_{1}\right)^{n}-2\left(\rho_{1}-\rho_{2}\right)^{n}+\left(t-\rho_{3}\right)^{n}\right], & \text { for } t \in\left[\rho_{3}, 1\right) .\end{cases}
$$

For HWC technique, the interval $[\alpha, \beta]$ is discretized using formula:

$$
t_{m}=\alpha+(\beta-\alpha) \frac{m-0.5}{2 M} .
$$

The Eq. (16) is known as collocation point (CP). Guass points (GPs) are defined as:

$$
G_{j}=h\left(\frac{j-1}{2}+\frac{3-\sqrt{3}}{6}\right), \quad G_{j+1}=h\left(\frac{j-1}{2}+\frac{3+\sqrt{3}}{6}\right) .
$$

Some of the recent work using HWC technique can be seen in [20-23].

\section{Numerical Scheme for Eight Order BVP}

In this section, HWC scheme is developed for solution of eight-order BVPs. For the [0, 1] interval, we developed the Haar wavelet collocation (HWC) method. The notation $\Theta=\sum_{i=1}^{N}$ is used. Let $u^{(8)}(t) \in L_{2}[0,1)$, then

$$
u^{(8)}(t)=\Theta a_{i} h_{i}(t)
$$

Integrating Eq. (17) from 0 to $t$ and using BCs we get the expressions of lower order derivatives and unknown function $u(t)$

$$
\begin{aligned}
& u^{(7)}(t)=u^{(7)}(0)+\Theta a_{i} p_{i, 1}(t), \\
& u^{(6)}(t)=u^{(6)}(0)+t u^{(7)}(0)+\Theta a_{i} p_{i, 2}(t), \\
& u^{(5)}(t)=u^{(5)}(0)+t u^{(6)}(0)+\frac{t^{2}}{2} u^{(7)}(0)+\Theta a_{i} p_{i, 3}(t), \\
& u^{(4)}(t)=u^{4}(0)+t u^{(5)}(0)+\frac{t^{2}}{2} u^{(6)}(0)+\frac{t^{3}}{6} u^{(7)}(0)+\Theta a_{i} p_{i, 4}(t), \\
& u^{(3)}(t)=\alpha_{4}+t u^{(4)}(0)+u^{(5)}(0) \frac{t^{2}}{2}+u^{(6)}(0) \frac{t^{3}}{6}+u^{(7)}(0) \frac{t^{4}}{24}+\Theta a_{i} p_{i, 5}(t), \\
& u^{(2)}(t)=\alpha_{3}+t \alpha_{4}+\frac{t^{2}}{2} u^{(4)}(0)+\frac{t^{3}}{6} u^{(5)}(0)+\frac{t^{4}}{24} u^{(6)}(0)+\frac{t^{5}}{120} u^{(7)}(0)+\Theta a_{i} p_{i, 6}(t), \\
& u^{(1)}(t)=\alpha_{2}+t \alpha_{3}+\frac{t^{2}}{2} \alpha_{4}+\frac{t^{3}}{6} u^{(4)}(0)+\frac{t^{4}}{24} u^{(5)}(0)+\frac{t^{5}}{120} u^{(6)}(0)+\frac{t^{6}}{720} u^{(7)}(0)+\Theta a_{i} p_{i, 7}(t),
\end{aligned}
$$

and

$$
\begin{aligned}
u(t)= & \alpha_{1}+t \alpha_{2}+\frac{t^{2}}{2} \alpha_{3}+\frac{t^{3}}{6} \alpha_{4}+\frac{t^{4}}{24} u^{(4)}(0)+\frac{t^{5}}{120} u^{(5)}(0) \\
& +\frac{t^{6}}{720} u^{(6)}(0)+\frac{t^{7}}{5040} u^{(7)}(0)+\Theta a_{i} p_{i, 8}(t)
\end{aligned}
$$


Now to find the unknown $u^{(4)}(0), u^{(5)}(0), u^{(6)}(0)$ and $u^{(7)}(0)$ in Eq. (25), we integrate Eqs. (21), (22), (23), (24) each from 0 to 1 and using BCs to obtain the following four equations:

$$
\begin{aligned}
& \beta_{4}-\alpha_{4}=u^{(4)}(0)+\frac{1}{2} u^{(5)}(0)+\frac{1}{6} u^{(6)}(0)+\frac{1}{24} u^{(7)}(0)+\Theta a_{i} c_{i, 4}, \\
& \beta_{3}-\alpha_{3}-\alpha_{4}=\frac{1}{2} u^{(4)}(0)+\frac{1}{6} u^{(5)}(0)+\frac{1}{24} u^{(6)}(0)+\frac{1}{120} u^{(7)}(0)+\Theta a_{i} c_{i, 5}, \\
& \beta_{2}-\alpha_{2}-\alpha_{3}-\frac{1}{2} \alpha_{4}=\frac{u^{(4)}(0)}{6}+\frac{u^{(5)}(0)}{24}+\frac{u^{(6)}(0)}{120}+\frac{u^{(7)}(0)}{720}+\Theta a_{i} c_{i, 6}, \\
& \beta_{1}-\alpha_{1}-\alpha_{2}-\frac{1}{2} \alpha_{3}-\frac{1}{6} \alpha_{4}=\frac{u^{(4)}(0)}{24}+\frac{u^{(5)}(0)}{120}+\frac{u^{(6)}(0)}{720}+\frac{u^{(7)}(0)}{5040}+\Theta a_{i} c_{i, 7}
\end{aligned}
$$

Finally solving these four Eqs. (26) to (29), we will get these unknown $u^{(7)}(0), u^{(6)}(0)$, $u^{(5)}(0)$ and $u^{(4)}(0)$ respectively

$$
\begin{aligned}
& u^{(4)}(0)=-4 \beta_{4}-16 \alpha_{4}+60 \beta_{3}-120 \alpha_{3}-360 \beta_{2}+3840 \alpha_{2}+840 \beta_{1}-840 \alpha_{1}+3 \Theta a_{i} c_{i, 4} \\
&-54 \Theta a_{i} c_{i, 5}+360 \Theta a_{i} c_{i, 6}-8340 \Theta a_{i} c_{i, 7}, \\
& u^{(5)}(0)=60 \beta_{4}- 840 \beta_{3}+1200 \alpha_{3}+120 \alpha_{4}+4680 \beta_{2}-20520 \alpha_{2}-10080 \beta_{1}+10080 \alpha_{1}-54 \Theta a_{i} c_{i, 4} \\
&+828 \Theta a_{i} c_{i, 5}-4680 \Theta a_{i} c_{i, 6}+10080 \Theta a_{i} c_{i, 7}, \\
& u^{(6)}(0)=- 360 \beta_{4}+4680 \beta_{3}-24480 \beta_{2}+25920 \alpha_{2}-5400 \alpha_{3}-480 \alpha_{4}+50400 \beta_{1}-50400 \alpha_{1} \\
&+ 360 \Theta a_{i} c_{i, 4}-4680 \Theta a_{i} c_{i, 5}+24480 \Theta a_{i} c_{i, 6}-50400 \Theta a_{i} c_{i, 7}
\end{aligned}
$$

and

$$
\begin{aligned}
u^{(7)}(0)= & 840 \beta_{4}-10080 \beta_{3}+50400 \beta_{2}-100800 \beta_{1}+10080 \alpha_{3}+50400 \alpha_{2}+840 \alpha_{4}+100800 \alpha_{1} \\
& -840 \Theta a_{i} c_{i, 4}+10080 \Theta a_{i} c_{i, 5}-50400 \Theta a_{i} c_{i, 6}+100800 \Theta a_{i} c_{i, 7} .
\end{aligned}
$$

Next we substitute the above values of Eqs. (30), (31), (32), (33) to Eqs. (21) and (25)

$$
\begin{aligned}
& u^{(4)}(t)=-4 \beta_{4}-16 \alpha_{4}+60 \beta_{3}-120 \alpha_{3}-360 \beta_{2}+3840 \alpha_{2}+840 \beta_{1}-840 \alpha_{1}+3 \Theta a_{i} c_{i, 4} \\
& -54 \Theta a_{i} c_{i, 5}+360 \Theta a_{i} c_{i, 6}-8340 \Theta a_{i} c_{i, 7}+t\left(60 \beta_{4}-840 \beta_{3}+1200 \alpha_{3}+120 \alpha_{4}+4680 \beta_{2}\right. \\
& \left.-20520 \alpha_{2}-10080 \beta_{1}+10080 \alpha_{1}-54 \Theta a_{i} c_{i, 4}\right)+\frac{t^{2}}{2}\left(-360 \beta_{4}+4680 \beta_{3}-24480 \beta_{2}+25920 \alpha_{2}\right. \\
& -5400 \alpha_{3}-480 \alpha_{4}+50400 \beta_{1}-50400 \alpha_{1}+360 \Theta a_{i} c_{i, 4}-4680 \Theta a_{i} c_{i, 5}+24480 \Theta a_{i} c_{i, 6} \\
& \left.-50400 \Theta a_{i} c_{i, 7}\right)+\frac{t^{3}}{6}\left(840 \beta_{4}-10080 \beta_{3}+50400 \beta_{2}-100800 \beta_{1}+10080 \alpha_{3}+50400 \alpha_{2}+840 \alpha_{4}\right. \\
& \left.+100800 \alpha_{1}-840 \Theta a_{i} c_{i, 4}+10080 \Theta a_{i} c_{i, 5}-50400 \Theta a_{i} c_{i, 6}+100800 \Theta a_{i} c_{i, 7}\right)+\Theta a_{i} p_{i, 4}(t),
\end{aligned}
$$

and

$$
\begin{aligned}
& u(t)=\alpha_{1}+t \alpha_{2}+\frac{t^{2}}{2} \alpha_{3}+\frac{t^{3}}{6} \alpha_{4}+\frac{t^{4}}{24}\left(-4 \beta_{4}-16 \alpha_{4}+60 \beta_{3}-120 \alpha_{3}-360 \beta_{2}+3840 \alpha_{2}+840 \beta_{1}\right. \\
& \left.-840 \alpha_{1}+3 \Theta a_{i} c_{i, 4}-54 \Theta a_{i} c_{i, 5}+360 \Theta a_{i} c_{i, 6}-8340 \Theta a_{i} c_{i, 7}\right)+\frac{t^{5}}{120}\left(60 \beta_{4}-840 \beta_{3}\right. \\
& +1200 \alpha_{3}+120 \alpha_{4}+4680 \beta_{2}-20520 \alpha_{2}-10080 \beta_{1}+10080 \alpha_{1}-54 \Theta a_{i} c_{i, 4}+828 \Theta a_{i} c_{i, 5} \\
& \left.-4680 \Theta a_{i} c_{i, 6}+10080 \Theta a_{i} c_{i, 7}\right)+\frac{t^{6}}{720}\left(-360 \beta_{4}+4680 \beta_{3}-24480 \beta_{2}+25920 \alpha_{2}-5400 \alpha_{3}\right. \\
& \left.-480 \alpha_{4}+50400 \beta_{1}-50400 \alpha_{1}+360 \Theta a_{i} c_{i, 4}-4680 \Theta a_{i} c_{i, 5}+24480 \Theta a_{i} c_{i, 6}-50400 \Theta a_{i} c_{i, 7}\right) \\
& +\frac{t^{7}}{5040}\left(840 \beta_{4}-10080 \beta_{3}+50400 \beta_{2}-100800 \beta_{1}+10080 \alpha_{3}+50400 \alpha_{2}+840 \alpha_{4}+100800 \alpha_{1}\right. \\
& \left.-840 \Theta a_{i} c_{i, 4}+10080 \Theta a_{i} c_{i, 5}-50400 \Theta a_{i} c_{i, 6}+100800 \Theta a_{i} c_{i, 7}\right)+\Theta a_{i} p_{i, 8}(t) .
\end{aligned}
$$




\section{Linear Case}

Substituting the values of $u^{(8)}(t), u^{(4)}(t)$ and $u(t)$ from Eqs. (17), (34), (35) to Eq. (1) and the collocation points and then simplification leads the following system of equations:

$$
\begin{aligned}
& \Theta a_{i}\left(h_{i}\left(t_{j}\right)+p_{i, 8}\left(t_{j}\right)+f\left(t_{j}\right)\left(\left(3-54 t_{j}+180 t_{j}^{2}-140 t_{j}^{3}\right) c_{i, 4}+\left(-54-828 t_{j}-2340 t_{j}^{2}+1680 t_{j}^{3}\right) c_{i, 5}\right.\right. \\
& \left.\left(360-4680 t_{j}+12240 t_{j}^{2}-8600 t_{j}^{3}\right) c_{i, 6}+\left(-8340+10080 t_{j}-25200 t_{j}^{2}+16800 t_{j}^{3}\right) c_{i, 7}+p_{i, 4}\left(t_{j}\right)\right) \\
& g\left(t_{j}\right)\left(\left(\frac{1}{8} t_{j}^{4}-\frac{9}{20} t_{j}^{5}+\frac{1}{2} t_{j}^{6}-\frac{7}{43} t_{j}^{7}\right) c_{i, 4}+\left(\frac{-9}{4} t_{j}^{4}+\frac{169}{10} t_{j}^{5}-39 t_{j}^{6}+14 t_{j}^{7}\right) c_{i, 5}+\left(15 t_{j}^{4}-39 t_{j}^{5}\right.\right. \\
& \left.\left.\left.+34 t_{j}^{6}-2 t_{j}^{7}\right) c_{i, 6}+\left(\frac{-695}{2} t_{j}^{4}+84 t_{j}^{5}-70 t_{j}^{6}+20 t_{j}^{7}\right) c_{i, 7}\right)\right)=h\left(t_{j}\right)-f\left(t_{j}\right)\left(\left(-4 \beta_{4}-16 \alpha_{4}+60 \beta_{3}\right.\right. \\
& \left.-120 \alpha_{3}-360 \beta_{2}+3840 \alpha_{2}+840 \beta_{1}-840 \alpha_{1}\right)+t_{j}\left(60 \beta_{4}-840 \beta_{3}+1200 \alpha_{3}+120 \alpha_{4}+4680 \beta_{2}\right. \\
& \left.-20520 \alpha_{2}-10080 \beta_{1}+10080 \alpha_{1}\right)+\frac{1}{2} t_{j}^{2}\left(-360 \beta_{4}+4680 \beta_{3}-24480 \beta_{2}+25920 \alpha_{2}-5400 \alpha_{3}-480 \alpha_{4}\right. \\
& \left.+50400 \beta_{1}-50400 \alpha_{1}\right)+\frac{1}{6} t_{j}^{3}\left(840 \beta_{4}-10080 \beta_{3}+50400 \beta_{2}-100800 \beta_{1}+10080 \alpha_{3}+50400 \alpha_{2}+840 \alpha_{4}\right. \\
& \left.\left.+100800 \alpha_{1}\right)\right)-g\left(t_{j}\right)\left(\alpha_{1}+t_{j} \alpha_{2}+\frac{t_{j}^{2}}{2} \alpha_{3}+\frac{t_{j}^{3}}{6} \alpha_{4}+\frac{t_{j}^{4}}{24}\left(-4 \beta_{4}-16 \alpha_{4}+60 \beta_{3}-120 \alpha_{3}-360 \beta_{2}+3840 \alpha_{2}\right.\right. \\
& \left.+840 \beta_{1}-840 \alpha_{1}\right)+\frac{t_{j}^{5}}{120}\left(60 \beta_{4}-840 \beta_{3}+1200 \alpha_{3}+120 \alpha_{4}+4680 \beta_{2}-20520 \alpha_{2}-10080 \beta_{1}+10080 \alpha_{1}\right) \\
& \left.+\frac{t_{j}^{6}}{720} u^{6}(0)+\frac{t_{j}^{7}}{5040}\left(840 \beta_{4}-10080 \beta_{3}+50400 \beta_{2}-100800 \beta_{1}+10080 \alpha_{3}+50400 \alpha_{2}+840 \alpha_{4}+100800 \alpha_{1}\right)\right)
\end{aligned}
$$

For the solution of this linear system of $N \times N$, the Gauss elimination approach is used. The solution provides the values of the $a i$ 's unknown coefficients. The solution is obtained by using these coefficients in Eq. (35).

\section{Nonlinear Case}

Substituting the values of $u(t), u^{(1)}(t), u^{(2)}(t), u^{(3)}(t), u^{(4)}(t), u^{(5)}(t), u^{(6)}(t), u^{(7)}(t)$, and $u^{(8)}(t)$ in $(1)$ and the collocation points, thus simplification leads:

$$
\begin{aligned}
& F\left(t, u, u^{(1)}, u^{(2)}, u^{(3)}, u^{(4)}, u^{(5)}, u^{(6)}, u^{(7)}\right)=\Theta a_{i} h_{i}\left(t_{j}\right)-F\left(t_{j},\right. \\
& \alpha_{1}+t_{j} \alpha_{2}+\frac{t_{j}^{2}}{2} \alpha_{3}+\frac{t_{j}^{3}}{6} \alpha_{4}+\frac{t_{j}^{4}}{24}\left(-4 \beta_{4}-16 \alpha_{4}+60 \beta_{3}-120 \alpha_{3}-360 \beta_{2}+3840 \alpha_{2}+840 \beta_{1}\right. \\
& \left.\quad-840 \alpha_{1}+3 \Theta a_{i} c_{i, 4}-54 \Theta a_{i} c_{i, 5}+360 \Theta a_{i} c_{i, 6}-8340 \Theta a_{i} c_{i, 7}\right)+\frac{t_{j}^{5}}{120}\left(60 \beta_{4}-840 \beta_{3}\right. \\
& \quad+1200 \alpha_{3}+120 \alpha_{4}+4680 \beta_{2}-20520 \alpha_{2}-10080 \beta_{1}+10080 \alpha_{1}-54 \Theta a_{i} c_{i, 4}+828 \Theta a_{i} c_{i, 5} \\
& \left.\quad-4680 \Theta a_{i} c_{i, 6}+10080 \Theta a_{i} c_{i, 7}\right)+\frac{t_{j}^{6}}{720}\left(-360 \beta_{4}+4680 \beta_{3}-24480 \beta_{2}+25920 \alpha_{2}-5400 \alpha_{3}\right. \\
& \left.\quad-480 \alpha_{4}+50400 \beta_{1}-50400 \alpha_{1}+360 \Theta a_{i} c_{i, 4}-4680 \Theta a_{i} c_{i, 5}+24480 \Theta a_{i} c_{i, 6}-50400 \Theta a_{i} c_{i, 7}\right) \\
& \quad+\frac{t_{j}^{7}}{5040}\left(840 \beta_{4}-10080 \beta_{3}+50400 \beta_{2}-100800 \beta_{1}+10080 \alpha_{3}+50400 \alpha_{2}+840 \alpha_{4}+100800 \alpha_{1}\right. \\
& \left.\quad-840 \Theta a_{i} c_{i, 4}+10080 \Theta a_{i} c_{i, 5}-50400 \Theta a_{i} c_{i, 6}+100800 \Theta a_{i} c_{i, 7}\right)+\Theta a_{i} p_{i, 8}\left(t_{j}\right), \\
& \alpha_{2}+t_{j} \alpha_{3}+\frac{t_{j}^{2}}{2} \alpha_{4}+\frac{t_{j}^{3}}{6}\left(-4 \beta_{4}-16 \alpha_{4}+60 \beta_{3}-120 \alpha_{3}-360 \beta_{2}+3840 \alpha_{2}+840 \beta_{1}-840 \alpha_{1}\right. \\
& \left.+3 \Theta a_{i} c_{i, 4}-54 \Theta a_{i} c_{i, 5}+360 \Theta a_{i} c_{i, 6}-8340 \Theta a_{i} c_{i, 7}\right)+\frac{t_{j}^{4}}{24}\left(60 \beta_{4}-840 \beta_{3}+1200 \alpha_{3}\right. \\
& +120 \alpha_{4}+4680 \beta_{2}-20520 \alpha_{2}-10080 \beta_{1}+10080 \alpha_{1}-54 \Theta a_{i} c_{i, 4}+828 \Theta a_{i} c_{i, 5}-4680 \Theta a_{i} c_{i, 6}
\end{aligned}
$$




$$
\begin{aligned}
& \left.+10080 \Theta a_{i} c_{i, 7}\right)+\frac{t_{j}^{5}}{120}\left(-360 \beta_{4}+4680 \beta_{3}-24480 \beta_{2}+25920 \alpha_{2}-5400 \alpha_{3}-480 \alpha_{4}+50400 \beta_{1}\right. \\
& \left.-50400 \alpha_{1}+360 \Theta a_{i} c_{i, 4}-4680 \Theta a_{i} c_{i, 5}+24480 \Theta a_{i} c_{i, 6}-50400 \Theta a_{i} c_{i, 7}\right)+\frac{t_{j}^{6}}{720}\left(840 \beta_{4}\right. \\
& -10080 \beta_{3}+50400 \beta_{2}-100800 \beta_{1}+10080 \alpha_{3}+50400 \alpha_{2}+840 \alpha_{4}+100800 \alpha_{1}-840 \Theta a_{i} c_{i, 4} \\
& \left.+10080 \Theta a_{i} c_{i, 5}-50400 \Theta a_{i} c_{i, 6}+100800 \Theta a_{i} c_{i, 7}\right)+\Theta a_{i} p_{i, 7}\left(t_{j}\right), \\
& \alpha_{3}+t_{j} \alpha_{4}+\frac{t_{j}^{2}}{2}\left(-4 \beta_{4}-16 \alpha_{4}+60 \beta_{3}-120 \alpha_{3}-360 \beta_{2}+3840 \alpha_{2}+840 \beta_{1}-840 \alpha_{1}+3 \Theta a_{i} c_{i, 4}\right. \\
& \left.-54 \Theta a_{i} c_{i, 5}+360 \Theta a_{i} c_{i, 6}-8340 \Theta a_{i} c_{i, 7}\right)+\frac{t_{j}^{3}}{6}\left(60 \beta_{4}-840 \beta_{3}+1200 \alpha_{3}+120 \alpha_{4}+4680 \beta_{2}\right. \\
& \left.-20520 \alpha_{2}-10080 \beta_{1}+10080 \alpha_{1}-54 \Theta a_{i} c_{i, 4}+828 \Theta a_{i} c_{i, 5}-4680 \Theta a_{i} c_{i, 6}+10080 \Theta a_{i} c_{i, 7}\right) \\
& +\frac{t_{j}^{4}}{24}\left(-360 \beta_{4}+4680 \beta_{3}-24480 \beta_{2}+25920 \alpha_{2}-5400 \alpha_{3}-480 \alpha_{4}+50400 \beta_{1}-50400 \alpha_{1}+360 \Theta a_{i} c_{i, 4}\right. \\
& \left.-4680 \Theta a_{i} c_{i, 5}+24480 \Theta a_{i} c_{i, 6}-50400 \Theta a_{i} c_{i, 7}\right)+\frac{t_{j}^{5}}{120}\left(840 \beta_{4}-10080 \beta_{3}+50400 \beta_{2}-100800 \beta_{1}\right. \\
& +10080 \alpha_{3}+50400 \alpha_{2}+840 \alpha_{4}+100800 \alpha_{1}-840 \Theta a_{i} c_{i, 4}+10080 \Theta a_{i} c_{i, 5}-50400 \Theta a_{i} c_{i, 6} \\
& \left.+100800 \Theta a_{i} c_{i, 7}\right)+\Theta a_{i} p_{i, 6}\left(t_{j}\right), \\
& \alpha_{4}+t_{j}\left(-4 \beta_{4}-16 \alpha_{4}+60 \beta_{3}-120 \alpha_{3}-360 \beta_{2}+3840 \alpha_{2}+840 \beta_{1}-840 \alpha_{1}+3 \Theta a_{i} c_{i, 4}-54 \Theta a_{i} c_{i, 5}\right. \\
& \left.+360 \Theta a_{i} c_{i, 6}-8340 \Theta a_{i} c_{i, 7}\right)+\frac{t_{j}^{2}}{2}\left(60 \beta_{4}-840 \beta_{3}+1200 \alpha_{3}+120 \alpha_{4}+4680 \beta_{2}-20520 \alpha_{2}-10080 \beta_{1}\right. \\
& \left.+10080 \alpha_{1}-54 \Theta a_{i} c_{i, 4}+828 \Theta a_{i} c_{i, 5}-4680 \Theta a_{i} c_{i, 6}+10080 \Theta a_{i} c_{i, 7}\right)+\frac{t_{j}^{3}}{6}\left(-360 \beta_{4}+4680 \beta_{3}\right. \\
& -24480 \beta_{2}+25920 \alpha_{2}-5400 \alpha_{3}-480 \alpha_{4}+50400 \beta_{1}-50400 \alpha_{1}+360 \Theta a_{i} c_{i, 4}-4680 \Theta a_{i} c_{i, 5} \\
& \left.+24480 \Theta a_{i} c_{i, 6}-50400 \Theta a_{i} c_{i, 7}\right)+\frac{t_{j}^{4}}{24}\left(840 \beta_{4}-10080 \beta_{3}+50400 \beta_{2}-100800 \beta_{1}+10080 \alpha_{3}\right. \\
& \left.+50400 \alpha_{2}+840 \alpha_{4}+100800 \alpha_{1}-840 \Theta a_{i} c_{i, 4}+10080 \Theta a_{i} c_{i, 5}-50400 \Theta a_{i} c_{i, 6}+100800 \Theta a_{i} c_{i, 7}\right) \\
& +\Theta a_{i} p_{i, 5}\left(t_{j}\right) \text {, } \\
& \left(\left(-4 \beta_{4}-16 \alpha_{4}+60 \beta_{3}-120 \alpha_{3}-360 \beta_{2}+3840 \alpha_{2}+840 \beta_{1}-840 \alpha_{1}+3 \Theta a_{i} c_{i, 4}-54 \Theta a_{i} c_{i, 5}\right.\right. \\
& \left.+360 \Theta a_{i} c_{i, 6}-8340 \Theta a_{i} c_{i, 7}\right)+t_{j}\left(60 \beta_{4}-840 \beta_{3}+1200 \alpha_{3}+120 \alpha_{4}+4680 \beta_{2}-20520 \alpha_{2}-10080 \beta_{1}\right. \\
& \left.+10080 \alpha_{1}-54 \Theta a_{i} c_{i, 4}+828 \Theta a_{i} c_{i, 5}-4680 \Theta a_{i} c_{i, 6}+10080 \Theta a_{i} c_{i, 7}\right)+\frac{t_{j}^{2}}{2}\left(-360 \beta_{4}+4680 \beta_{3}\right. \\
& -24480 \beta_{2}+25920 \alpha_{2}-5400 \alpha_{3}-480 \alpha_{4}+50400 \beta_{1}-50400 \alpha_{1}+360 \Theta a_{i} c_{i, 4}-4680 \Theta a_{i} c_{i, 5} \\
& \left.+24480 \Theta a_{i} c_{i, 6}-50400 \Theta a_{i} c_{i, 7}\right)+\frac{t_{j}^{3}}{6}\left(840 \beta_{4}-10080 \beta_{3}+50400 \beta_{2}-100800 \beta_{1}+10080 \alpha_{3}\right. \\
& \left.+50400 \alpha_{2}+840 \alpha_{4}+100800 \alpha_{1}-840 \Theta a_{i} c_{i, 4}+10080 \Theta a_{i} c_{i, 5}-50400 \Theta a_{i} c_{i, 6}+100800 \Theta a_{i} c_{i, 7}\right) \\
& +\Theta a_{i} p_{i, 4}\left(t_{j}\right), \\
& \left(60 \beta_{4}-840 \beta_{3}+1200 \alpha_{3}+120 \alpha_{4}+4680 \beta_{2}-20520 \alpha_{2}-10080 \beta_{1}+10080 \alpha_{1}-54 \Theta a_{i} c_{i, 4}\right. \\
& \left.+828 \Theta a_{i} c_{i, 5}-4680 \Theta a_{i} c_{i, 6}+10080 \Theta a_{i} c_{i, 7}\right)+t_{j}\left(-360 \beta_{4}+4680 \beta_{3}-24480 \beta_{2}+25920 \alpha_{2}\right. \\
& -5400 \alpha_{3}-480 \alpha_{4}+50400 \beta_{1}-50400 \alpha_{1}+360 \Theta a_{i} c_{i, 4}-4680 \Theta a_{i} c_{i, 5}+24480 \Theta a_{i} c_{i, 6} \\
& \left.-50400 \Theta a_{i} c_{i, 7}\right)+\frac{t_{j}^{2}}{2}\left(840 \beta_{4}-10080 \beta_{3}+50400 \beta_{2}-100800 \beta_{1}+10080 \alpha_{3}+50400 \alpha_{2}+840 \alpha_{4}\right. \\
& \left.+100800 \alpha_{1}-840 \Theta a_{i} c_{i, 4}+10080 \Theta a_{i} c_{i, 5}-50400 \Theta a_{i} c_{i, 6}+100800 \Theta a_{i} c_{i, 7}\right)+\Theta a_{i} p_{i, 3}\left(t_{j}\right), \\
& \left(-360 \beta_{4}+4680 \beta_{3}-24480 \beta_{2}+25920 \alpha_{2}-5400 \alpha_{3}-480 \alpha_{4}+50400 \beta_{1}-50400 \alpha_{1}+360 \Theta a_{i} c_{i, 4}\right. \\
& \left.-4680 \Theta a_{i} c_{i, 5}+24480 \Theta a_{i} c_{i, 6}-50400 \Theta a_{i} c_{i, 7}\right)+t_{j}\left(840 \beta_{4}-10080 \beta_{3}+50400 \beta_{2}-100800 \beta_{1}\right. \\
& +10080 \alpha_{3}+50400 \alpha_{2}+840 \alpha_{4}+100800 \alpha_{1}-840 \Theta a_{i} c_{i, 4}+10080 \Theta a_{i} c_{i, 5}-50400 \Theta a_{i} c_{i, 6} \\
& \left.+100800 \Theta a_{i} c_{i, 7}\right)+\Theta a_{i} p_{i, 2}\left(t_{j}\right) \text {, }
\end{aligned}
$$




$$
\begin{aligned}
& \left(840 \beta_{4}-10080 \beta_{3}+50400 \beta_{2}-100800 \beta_{1}+10080 \alpha_{3}+50400 \alpha_{2}+840 \alpha_{4}+100800 \alpha_{1}-840 \Theta a_{i} c_{i, 4}\right. \\
& \left.+10080 \Theta a_{i} c_{i, 5}-50400 \Theta a_{i} c_{i, 6}+100800 \Theta a_{i} c_{i, 7}\right)+\Theta a_{i} p_{i, 1}\left(t_{j}\right) .
\end{aligned}
$$

The Jacobian is:

$$
\begin{aligned}
& \frac{\partial}{\partial a_{m}}\left(F_{j}\left(a_{1}, a_{2}, \ldots \ldots, a_{N}\right)\right) \\
& =h_{m}\left(t_{j}\right)-\frac{t_{j}^{4}}{24}\left(3 c_{m, 4}-54 c_{m, 5}+360 c_{m, 6}-8340 c_{m, 7}\right)-\frac{t_{j}^{5}}{120}\left(-54 c_{m, 4}+828 c_{m, 5}-4680 c_{m, 6}+10080 c_{m, 7}\right) \\
& -\frac{t_{j}^{6}}{720}\left(360 c_{m, 4}-4680 c_{m, 5}+24480 c_{m, 6}-50400 c_{m, 7}\right)-\frac{t_{j}^{7}}{5040}\left(-840 c_{m, 4}+10080 c_{m, 5}-50400 c_{m, 6}\right. \\
& \left.+100800 c_{m, 7}\right)-p_{m, 8}\left(t_{j}\right)-\frac{t_{j}^{3}}{6}\left(3 c_{m, 4}-54 c_{m, 5}+360 c_{m, 6}-8340 c_{m, 7}\right)-\frac{t_{j}^{4}}{24}\left(-54 c_{m, 4}+828 c_{m, 5}-4680 c_{m, 6}\right. \\
& \left.+10080 c_{m, 7}\right)-\frac{t_{j}^{5}}{120}\left(360 c_{m, 4}-4680 c_{m, 5}+24480 c_{m, 6}-50400 c_{m, 7}\right)-\frac{t_{j}^{6}}{720}\left(-840 c_{m, 4}+10080 c_{m, 5}\right. \\
& \left.-50400 c_{m, 6}+100800 c_{m, 7}\right)-p_{m, 7}(t)-\frac{t_{j}^{2}}{2}\left(3 c_{m, 4}-54 c_{m, 5}+360 c_{m, 6}-8340 c_{m, 7}\right)-\frac{t_{j}^{3}}{6}\left(-54 c_{m, 4}\right. \\
& \left.+828 c_{m, 5}-4680 c_{m, 6}+10080 c_{m, 7}\right)-\frac{t_{j}^{4}}{24}\left(360 c_{m, 4}-4680 c_{m, 5}+24480 c_{m, 6}-50400 c_{m, 7}\right)-\frac{t_{j}^{5}}{120}\left(-840 c_{m, 4}(38\right. \\
& \left.+10080 c_{m, 5}-50400 c_{m, 6}+100800 c_{m, 7}\right)-p_{m, 6}(t)-t_{j}\left(3 c_{m, 4}-54 c_{m, 5}+360 c_{m, 6}-8340 c_{m, 7}\right)-\frac{t_{j}^{2}}{2}\left(-54 c_{m, 4}\right. \\
& \left.+828 c_{m, 5}-4680 c_{m, 6}+10080 c_{m, 7}\right)-\frac{t_{j}^{3}}{6}\left(360 c_{m, 4}-4680 c_{m, 5}+24480 c_{m, 6}-50400 c_{m, 7}\right)-\frac{t_{j}^{4}}{24}\left(-840 c_{m, 4}\right. \\
& \left.+10080 c_{m, 5}-50400 c_{m, 6}+100800 c_{m, 7}\right)-p_{m, 5}(t)-\left(3 c_{m, 4}-54 c_{m, 5}+360 c_{m, 6}-8340 c_{m, 7}\right)-t_{j}\left(-54 c_{m, 4}\right. \\
& \left.+828 c_{m, 5}-4680 c_{m, 6}+10080 c_{m, 7}\right)-\frac{t_{j}^{2}}{2}\left(360 c_{m, 4}-4680 c_{m, 5}+24480 c_{m, 6}-50400 c_{m, 7}\right)-\frac{t_{j}^{3}}{6}\left(-840 c_{m, 4}\right. \\
& \left.+10080 c_{m, 5}-50400 c_{m, 6}+100800 c_{m, 7}\right)-p_{m, 4}(t)-\left(-54 c_{m, 4}+828 c_{m, 5}-4680 c_{m, 6}+10080 c_{m, 7}\right) \\
& -t_{j}\left(360 c_{m, 4}-4680 c_{m, 5}+24480 c_{m, 6}-50400 c_{m, 7}\right)-\frac{t_{j}^{2}}{2}\left(-840 c_{m, 4}+10080 c_{m, 5}-50400 c_{m, 6}+100800 c_{m, 7}\right) \\
& -p_{m, 3}(t)-\left(360 c_{m, 4}-4680 c_{m, 5}+24480 c_{m, 6}-50400 c_{m, 7}\right)-t_{j}\left(-840 c_{m, 4}+10080 c_{m, 5}-50400 c_{m, 6}\right. \\
& \left.+100800 c_{m, 7}\right)-p_{m, 2}(t)-\left(-840 c_{m, 4}+10080 c_{m, 5}-50400 c_{m, 6}+100800 c_{m, 7}\right)-p_{m, 1}\left(t_{j}\right) .
\end{aligned}
$$

For the solution of this nonlinear $N \times N$ scheme of algebraic equations, Broyden's method is used. The solution provides the values of the unknown coefficients of Haar ai's. Using these unknown coefficients ai's in Eq. (35) the approximate solution at CPs is obtained.

\section{Convergence analysis}

Lemma 3.1 Let $u(\eta) \in L_{2}(\mathbb{R})$ such that the first order derivative on open interval $(0,1)$ is bounded, then error norm at $J^{\text {th }}$ level has the following inequality

$$
\left\|e_{j}(\eta)\right\|=\sqrt{\frac{K}{7}} C 2^{-(3) 2^{J-1}},
$$

where $C, K$ are constants and $M$ is a positive number related to $J^{\text {th }}$ resolution of the wavelet defined earlier.

Proof: See [24].

\section{Numerical Examples}

Some examples are given in this section to show the performance of the HWC technique. By using the proposed HWC algorithm, three linear and two nonlinear eight-order BVPs 


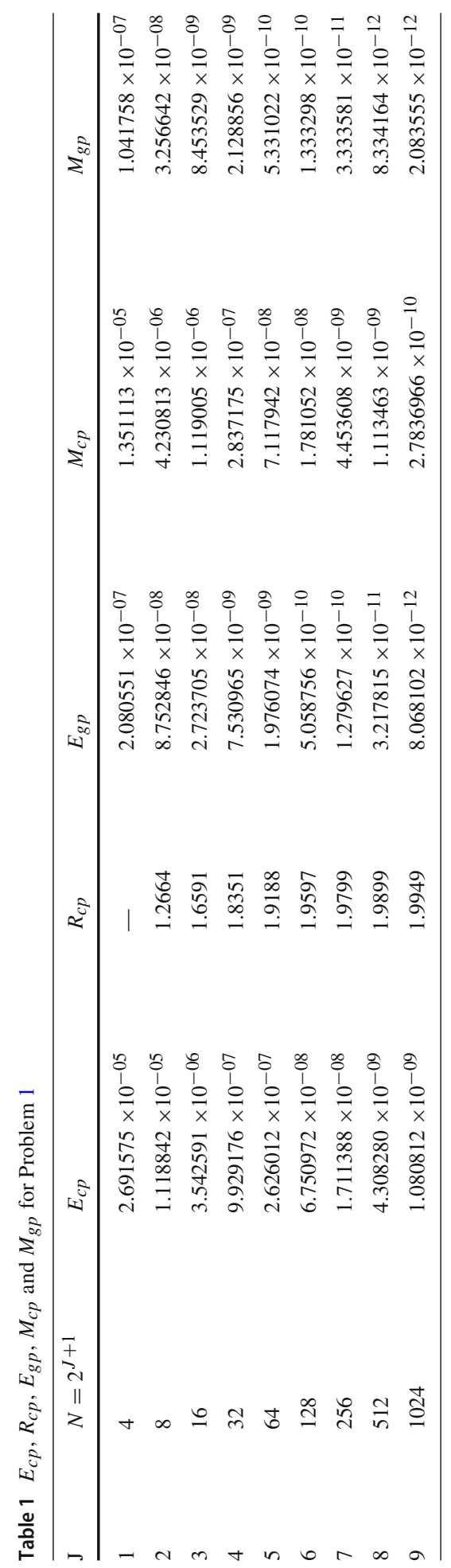


are being evaluated. If $u a p$ is approximate and uex is the exact solution for CPs and GPs respectively, then $E_{c p}$ and $E_{g p}$ are specified as the maximum absolute errors:

$$
\begin{aligned}
& E_{c p}=\max \left|u_{\text {exc }}-u_{\text {apc }}\right| \\
& E_{g p}=\max \left|u_{\text {exg }}-u_{\text {apg }}\right| .
\end{aligned}
$$

The $M_{c p}$ and $M_{g p}$ Root mean square root errors at CPs and GPs are:

$$
\begin{aligned}
M_{c p} & =\sqrt{\frac{1}{N}\left(\Theta\left|u_{e x c}-u_{a p c}\right|^{2}\right)} \\
M_{g p} & =\sqrt{\frac{1}{N}\left(\Theta\left|u_{e x g}-u_{\text {apg }}\right|^{2}\right)} .
\end{aligned}
$$

At CPs, the convergence rate is denoted by $R c p$ and is:

$$
R_{c p}=\frac{\log \left[u_{a p c}(N / 2) / u_{a p c}(N)\right]}{\log 2} .
$$

Problem 1 Consider the eight order BVP [25]

$$
\left\{\begin{array}{l}
u^{(8)}(t)+t u(t)=-\left(48+15 t+t^{3}\right) e^{t}, \quad t \in[0,1] \\
u(0)=0, \quad u(1)=0, \quad u^{(1)}(0)=1 \\
u^{(1)}(1)=-e, \quad u^{(2)}(0)=0, \quad u^{(2)}(1)=-4 e \\
u^{(3)}(0)=-3, \quad u^{(3)}(1)=-9 e
\end{array}\right.
$$

The analytical solution is

$$
u(t)=t(1-t) e^{t}
$$

The maximum absolute values of errors of Reproducing kernel technique [25] is $1.63 \times$ $10^{-10}$, non-polynomial spline technique [25] is $5.623 \times 10^{-09}$, Inc and Evans [26] results is $3.73 \times 10^{-09}$, variational iteration technique [27] is $1.10 \times 10^{-08}$, while the results of our method is decreased up to order $10^{-12}$. It is found from Table 1 that HWC results are better than other techniques. The approximate and analytical solution comparison is given in Fig. 1.

Problem 2 Consider the eight-order BVP [25]

$$
\left\{\begin{array}{l}
u^{(6)}(t)-u(t)=-8(7 \sin t+2 t \cos t) e^{t}, \quad-1 \leq t \leq 1 \\
u(-1)=u(1)=0, \quad u^{(1)}(-1)=u^{(1)}(1)=2 \sin 1, \\
u^{(2)}(-1)=-u^{(2)}(1)=-4 \cos 1-2 \sin 1, \quad u^{(3)}(-1)=-u^{(3)}(1)=6 \cos 1-6 \sin 1 .
\end{array}\right.
$$

The exact solution is

$$
u(t)=\left(t^{2}-1\right) \sin t
$$

It is noted that the absolute value errors are better than the [25] Siddiqi and Ghazala [28] and Siddiqi and Twizell [6] reproduction kernel techniques as shown in Table 2. The comparison of maximum absolute relative errors are shown in Table 3. Comparison of numerical and analytical solution for 32 CPs of Problem 2 are shown in Fig. 2. 


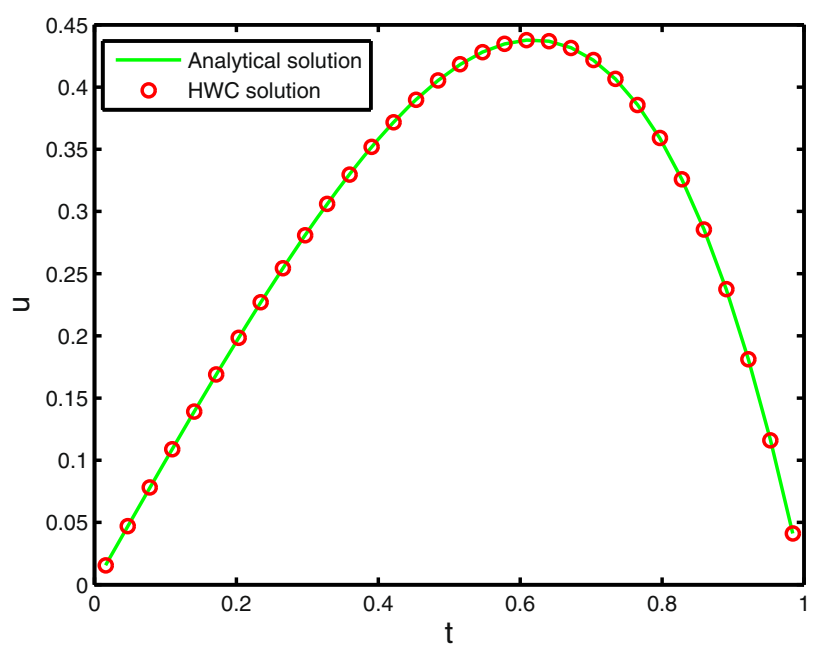

Fig. 1 Comparison of numerical and analytical solution for 32 discrete CPs of Problem 1

Problem 3 Consider the nonlinear eight order BVP [25]

$$
\left\{\begin{array}{l}
u^{(8)}(t)=e^{-t} u^{2}(t) \quad t \in[0,1] \\
u(0)=u^{(2)}(0)=u^{(4)}(0)=u^{(6)}(0)=1 \\
u(1)=u^{(2)}(1)=u^{(4)}(1)=u^{(6)}(1)=e .
\end{array}\right.
$$

The exact solution is

$$
u(t)=e^{t}
$$

It is clear that the absolute value errors are higher than those of [25], Mestrovic [29], He [30] and Noor and Mohyud-Din [31] kernel reproduction techniques as shown in Table 4. Figure 3 represent the comparison of exact and approximate solution for 32 CPs (Table 5).

Problem 4 Consider the nonlinear eight order BVP [25]

$$
\left\{\begin{array}{l}
u^{(8)}(t)+t(1-t) u^{(4)}(t)+e^{u(t)} u(t)+u^{3}(t)=f(t, u(t)), t \in[0,1], \\
u(0)=1, \quad u^{(2)}(0)=3, \quad u^{(4)}(0)=13, \quad u^{(6)}(0)=31, \\
u(1)=2 e, \quad u^{(2)}(1)=8 e, \quad u^{(4)}(1)=22 e, \quad u^{(6)}(1)=44 e,
\end{array}\right.
$$

where

$$
f(t, u(t))=e^{t}\left(57+e^{e^{t}\left(1+t^{2}\right)}\left(1+t^{2}\right)+e^{2 t}\left(1+t^{2}\right)^{3}-t(-29+t(4+t(7+t)))\right)(48)
$$

The analytical solution is

$$
u(t)=e^{t}\left(1+t^{2}\right)
$$

The $E_{c p}, R_{c p}, E_{g p}, M_{c p}$ and $M_{g p}$ errors for Problem 4 are shown in Table 5. Comparison of numerical and analytical solution for 32 CPs of Problem 4 are shown in Fig. 4. 


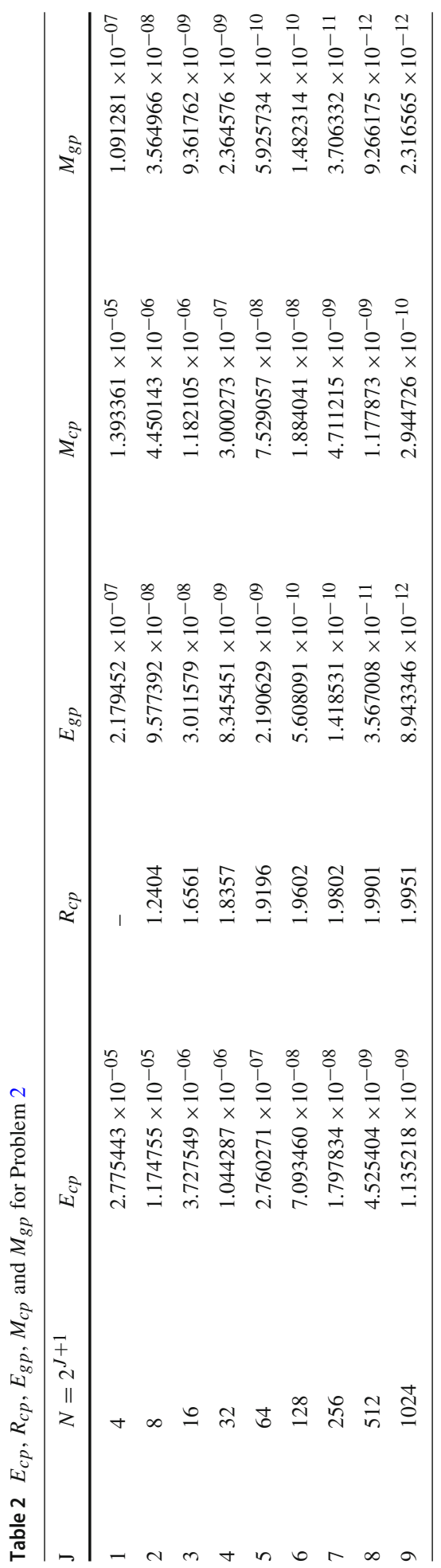


Table 3 Maximum absolute relative errors

\begin{tabular}{llll}
\hline $\begin{array}{l}\text { The technique developed } \\
\text { in this paper }\end{array}$ & $\begin{array}{l}\text { Reproducing kernel } \\
\text { space technique [25] }\end{array}$ & $\begin{array}{l}\text { variational iteration } \\
\text { method [28] }\end{array}$ & Spline technique [6] \\
\hline $2.47 \times 10^{-11}$ & $4.90 \times 10^{-09}$ & $1.20 \times 10^{-05}$ & $1.02 \times 10^{-08}$ \\
\hline
\end{tabular}

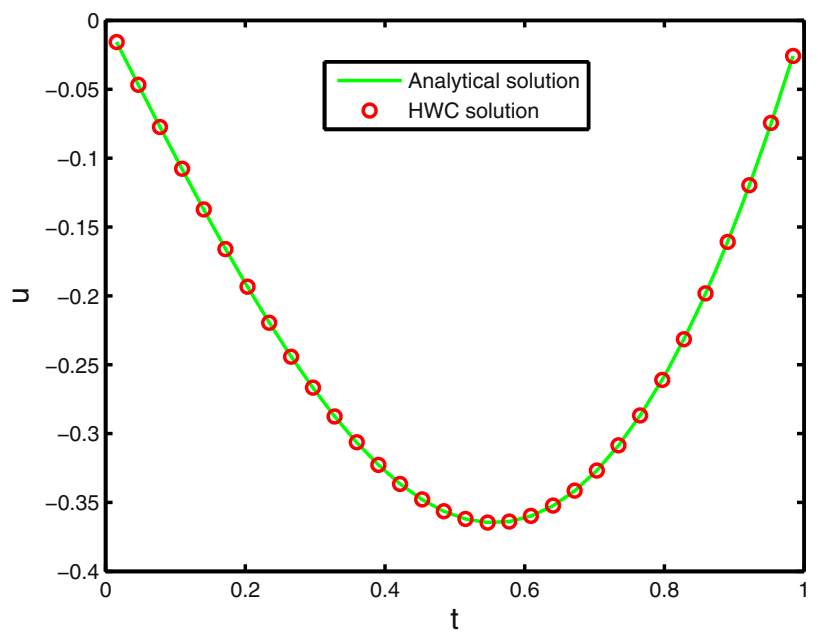

Fig. 2 Comparison of numerical and analytical solution for 32 discrete CPs of Problem 2

Table $4 E_{c p}, R_{c p}, E_{g p}, M_{c p}$ and $M_{g p}$ for Problem 3

\begin{tabular}{lllllll}
\hline $\mathrm{J}$ & $N=2^{J+1}$ & $E_{c p}$ & $R_{c p}$ & $E_{g p}$ & $M_{c p}$ & $M_{g p}$ \\
\hline 1 & 4 & $4.173104 \times 10^{-07}$ & - & $3.235788 \times 10^{-09}$ & $2.094854 \times 10^{-07}$ & $1.620200 \times 10^{-09}$ \\
2 & 8 & $1.740778 \times 10^{-07}$ & 1.2614 & $1.373027 \times 10^{-09}$ & $6.584904 \times 10^{-08}$ & $5.109014 \times 10^{-10}$ \\
3 & 16 & $5.514157 \times 10^{-08}$ & 1.6585 & $4.281679 \times 10^{-10}$ & $1.743127 \times 10^{-08}$ & $1.329322 \times 10^{-10}$ \\
4 & 32 & $1.545372 \times 10^{-08}$ & 1.8352 & $1.184415 \times 10^{-10}$ & $4.420518 \times 10^{-09}$ & $3.349678 \times 10^{-11}$ \\
5 & 64 & $4.086644 \times 10^{-09}$ & 1.9189 & $3.108047 \times 10^{-11}$ & $1.109082 \times 10^{-09}$ & $8.389443 \times 10^{-12}$ \\
6 & 128 & $1.050519 \times 10^{-09}$ & 1.9598 & $7.956746 \times 10^{-12}$ & $2.775180 \times 10^{-10}$ & $2.098303 \times 10^{-12}$ \\
7 & 256 & $2.662985 \times 10^{-10}$ & 1.9799 & $2.012834 \times 10^{-12}$ & $6.939491 \times 10^{-11}$ & $5.246569 \times 10^{-13}$ \\
\hline
\end{tabular}

Problem 5 Consider the eight order BVP [25]

$$
\left\{\begin{array}{l}
u^{(8)}(t)=-8 e^{t}+u(t), \\
u(0)=1, \quad u^{(1)}(0)=0, \quad u^{(2)}(0)=-1, \quad u^{(3)}(0)=-2, \\
u^{(4)}(0)=-3, \quad u^{(5)}(1)=-4, \quad u^{(1)}(1)=-e, \quad u^{(2)}(1)=-2 e .
\end{array} t \in[0,1],\right.
$$

The analytical solution is

$$
u(t)=(1-t) e^{t} .
$$

It is noted that the absolute value errors are higher than those of the [25] kernel reproduction technique, the [30] technique and the [32] technique of Golbabai and Javidi, as shown 


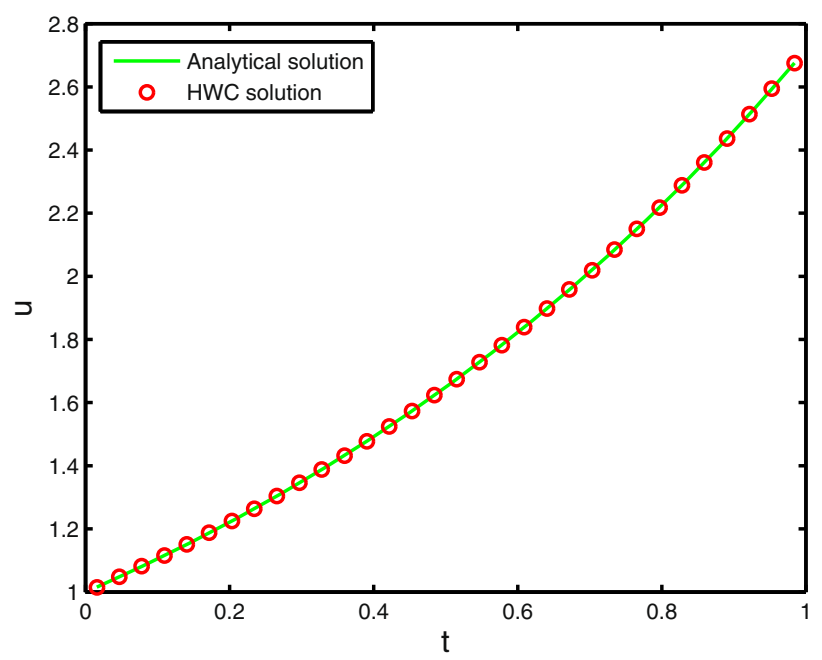

Fig. 3 Comparison of numerical and analytical solution for 32 discrete CPs of Problem 3

Table $5 E_{c p}, R_{c p}, E_{g p}, M_{c p}$ and $M_{g p}$ for Problem 4

\begin{tabular}{lllllll}
\hline $\mathrm{J}$ & $N=2^{J+1}$ & $E_{c p}$ & $R_{c p}$ & $E_{g p}$ & $M_{c p}$ & $M_{g p}$ \\
\hline 1 & 4 & $3.112471 \times 10^{-05}$ & - & $2.406584 \times 10^{-07}$ & $1.562397 \times 10^{-05}$ & $1.205006 \times 10^{-07}$ \\
2 & 8 & $1.294180 \times 10^{-05}$ & 1.2660 & $1.013188 \times 10^{-07}$ & $4.893994 \times 10^{-06}$ & $3.769762 \times 10^{-08}$ \\
3 & 16 & $4.097900 \times 10^{-06}$ & 1.6591 & $3.153405 \times 10^{-08}$ & $1.294502 \times 10^{-06}$ & $9.787451 \times 10^{-09}$ \\
4 & 32 & $1.148549 \times 10^{-06}$ & 1.8351 & $8.719413 \times 10^{-09}$ & $3.282196 \times 10^{-07}$ & $2.464907 \times 10^{-09}$ \\
5 & 64 & $3.037585 \times 10^{-07}$ & 1.9188 & $2.287929 \times 10^{-09}$ & $8.234451 \times 10^{-08}$ & $6.172633 \times 10^{-10}$ \\
6 & 128 & $7.808993 \times 10^{-08}$ & 1.9597 & $5.857106 \times 10^{-10}$ & $2.060427 \times 10^{-08}$ & $1.543791 \times 10^{-10}$ \\
7 & 256 & $1.979592 \times 10^{-08}$ & 1.9799 & $1.481575 \times 10^{-10}$ & $5.152201 \times 10^{-09}$ & $3.859870 \times 10^{-11}$ \\
\hline
\end{tabular}

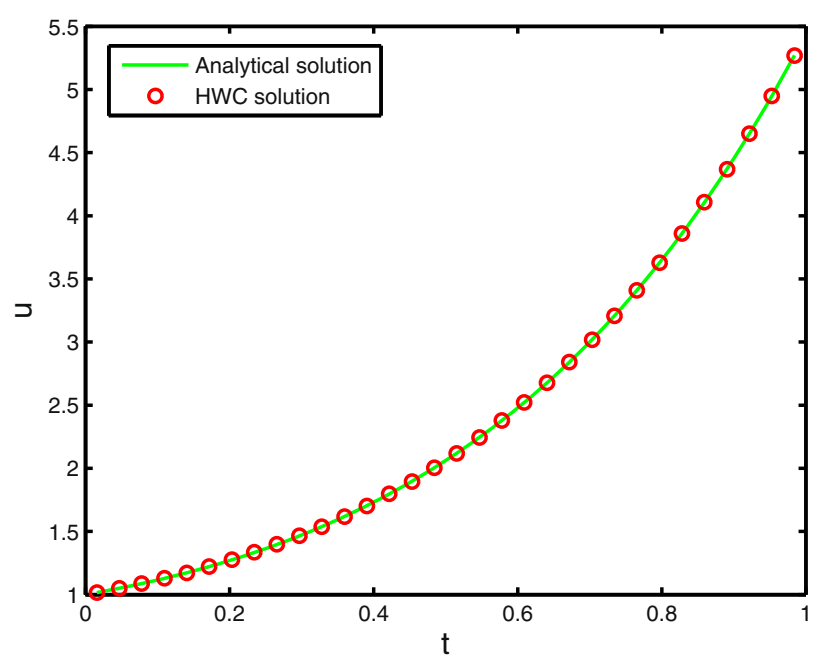

Fig. 4 Comparison of numerical and analytical solution for 32 discrete CPs of Problem 4 
Table $6 E_{c p}, R_{c p}, E_{g p}, M_{c p}$ and $M_{g p}$ for Problem 5

\begin{tabular}{lllllll}
\hline $\mathrm{J}$ & $N=2^{J+1}$ & $E_{c p}$ & $R_{c p}$ & $E_{g p}$ & $M_{c p}$ & $M_{g p}$ \\
\hline 1 & 4 & $3.374919 \times 10^{-06}$ & - & $2.613212 \times 10^{-08}$ & $1.694157 \times 10^{-06}$ & $1.308468 \times 10^{-08}$ \\
2 & 8 & $1.405496 \times 10^{-06}$ & 1.2638 & $1.104438 \times 10^{-08}$ & $5.315777 \times 10^{-07}$ & $4.109436 \times 10^{-09}$ \\
3 & 16 & $4.451187 \times 10^{-07}$ & 1.6588 & $3.440691 \times 10^{-09}$ & $1.406601 \times 10^{-07}$ & $1.068066 \times 10^{-09}$ \\
4 & 32 & $1.24752 \times 10^{-07}$ & 1.8351 & $9.515733 \times 10^{-10}$ & $3.566748 \times 10^{-08}$ & $2.690592 \times 10^{-10}$ \\
5 & 64 & $3.299148 \times 10^{-08}$ & 1.9189 & $2.496969 \times 10^{-10}$ & $8.948546 \times 10^{-09}$ & $6.738261 \times 10^{-11}$ \\
6 & 128 & $8.481130 \times 10^{-09}$ & 1.9598 & $6.392287 \times 10^{-11}$ & $2.239121 \times 10^{-09}$ & $1.685286 \times 10^{-11}$ \\
7 & 256 & $2.149939 \times 10^{-09}$ & 1.9799 & $1.616929 \times 10^{-11}$ & $5.599042 \times 10^{-10}$ & $4.213647 \times 10^{-12}$ \\
8 & 512 & $5.412233 \times 10^{-10}$ & 1.9899 & $4.065969 \times 10^{-12}$ & $1.399838 \times 10^{-10}$ & $1.053435 \times 10^{-12}$ \\
9 & 1024 & $1.357751 \times 10^{-10}$ & 1.9950 & $1.019518 \times 10^{-12}$ & $3.499644 \times 10^{-11}$ & $2.633669 \times 10^{-13}$ \\
\hline
\end{tabular}

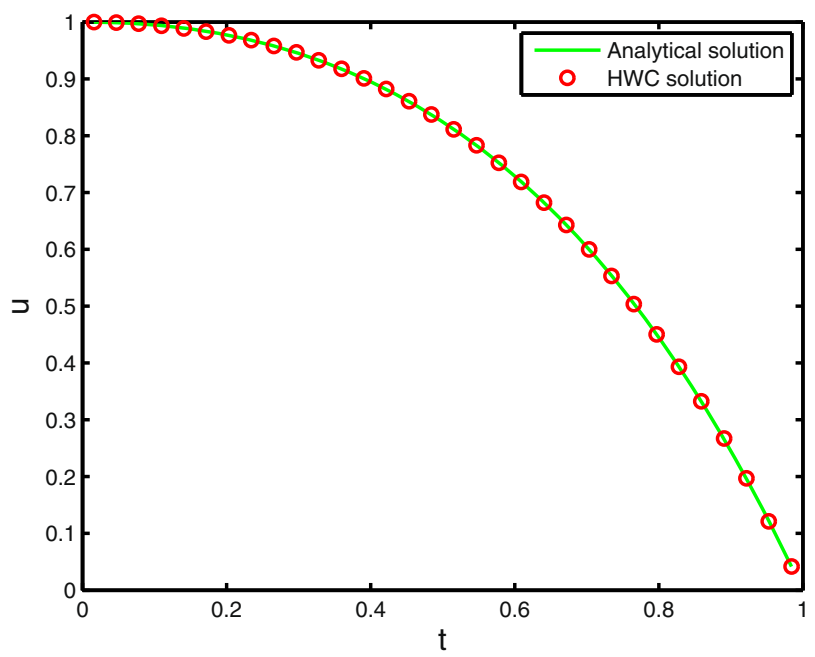

Fig. 5 Comparison of numerical and analytical solution for 32 discrete CPs of Problem 5

in Table 6. Comparison of numerical and analytical solution for 32 CPs of Problem 5 are shown in Fig. 5.

\section{Conclusion}

In this paper, the HWC technique is utilized to find the solution of linear and nonlinear eight order BVPs. The Haar technique is utilized for solution of eight order BVPs. The maximum absolute, mean square root errors and convergence rate for distinct number of discrete CPs are shown in tables. The obtained results are also compared with some other techniques available in literature. The Haar technique can be applied to similar problems that arise in physics and engineering or an any applied science. In figures for each linear and nonlinear problems, the comparison of exact and Haar solution is also shown. The errors in absolute values were found to be better than those compared with [25,29-32]. 


\section{Compliance with ethical standards}

Conflicts of interest There exists no conflict of interest.

\section{References}

1. Chandrasekhar, S.: Hydrodynamic and Hydromagnetic Stability. Clarendon Press, Oxford (1961)

2. Bishop, R.E.D., Cannon, S.M., Miao, S.: On coupled bending and torsional vibration of uni-form beams. J. Sound Vib. 131, 309-325 (1989)

3. Akram, G., Siddiqi, S.: Nonic spline solutions of eighth order boundary value problems Appl. Math. Comput. 182, 829-845 (2006)

4. Siddiqi, S., Akram, G.: Solution of eighth-order boundary value problems using the non-polynomial spline technique. Int. J. Comput. Math. 182, 347-368 (2007)

5. Wazwaz, A.M.: The numerical solutions of special eighth-order boundary value problems by the modified decomposition method. Neural Parallel Sci. Comput. 8, 133-146 (2000)

6. Siddiqi, S.S., Twizell, E.H.: Spline solutions of linear eighth-order boundary-value problems. Comput. Methods Appl. Mech. Eng. 131, 457-464 (1996)

7. Asif, M., Haider, N., Al-Mdallal, Q.M., Khan, I.: A Haar wavelet collocation approach for solving one and two-dimensional second-order linear and nonlinear hyperbolic telegraph equations. Numer. Methods Partial Differ. Equ. 36(6), 1962-1981 (2020)

8. Abdeljawad, T., Amin, R., Shah, K., Al-Mdallal, Q.M., Jarad, F.: Efficient sustainable algorithm for numerical solutions of systems of fractional order differential equations by Haar wavelet collocation method. Alex. Eng. J. 59(4), 2391-2400 (2020)

9. Asif, M., Khan, I., Haider, N., Al-Mdallal, Q.M.: Legendre multi-wavelets collocation method for numerical solution of linear and nonlinear integral equations. Alex. Eng. J. 59(6), 5099-5109 (2020)

10. Boutayeb, A., Twizell, E.H.: Finite-difference methods for the solution of special eighth-order boundaryvalue problems. Int. J. Comput. Math. 48, 63-75 (1993)

11. Siddiqi, S.S., Twizell, E.H.: Spline solutions of linear twelfth-order boundary-value problems. Comput. J. Comput. Appl. Math. 78, 371-390 (1997)

12. Wu, T.Y., Liu, G.R.: The differential quadrature as a numerical method to solve the differential equation. Comput. Mech. 24, 197-205 (1999)

13. Wu, T.Y., Liu, G.R.: A generalized differential quadrature rule for initial-value differential equations. J. Sound Vib. 233, 195-213 (2000)

14. Wu, T.Y., Liu, G.R.: The generalized differential quadrature rule for fourth-order differential equations. Int. J. Numer. Methods Eng. 50, 1907-1929 (2001)

15. Wu, T.Y., Liu, G.R.: Application of the generalized differential quadrature rule to sixth-order differential equations. Commun. Numer. Methods Eng. 16, 777-784 (2000)

16. Liu, G.R., Wu, T.Y.: Differential quadrature solutions of eighth-order boundary-value differential equations. J. Comput. Appl. Math. 145, 223-235 (2002)

17. Sher, M., Shah, K., Khan, Z.A., Khan, H., Khan, A.: Computational and theoretical modeling of the transmission dynamics of novel COVID-19 under Mittag-Leffler power law. Alex. Eng. J. 59(5), 31333147 (2020)

18. Khan, A., Gomez-Aguilar, J.F., Abdeljawad, T., Khan, H.: Stability and numerical simulation of a fractional order plant nectar pollinator model, Alex. Eng. J., 59, 49-59 (2020). solutions of fractional order HIV/AIDS model, Chaos, Solitons Fractals, 122, 119-128 (2019)

19. Aziz, I., Amin, R.: Numerical solution of a class of delay differential and delay partial differential equations via haar wavelet. Appl. Math. Model. 40, 10286-10299 (2016)

20. Reisenhofer, R., Bosse, S., Kutyniok, G., Wiegand, T.: A Haar wavelet-based perceptual similarity index for image quality assessment. Signal Process Image Commun 61, 33-43 (2018)

21. Adiguna, B.J., Buchanb, A.G., Adama, A., Dargavillea, S., Goffina, M.A.: A Haar wavelet method for angularly discretising the boltzmann transport equation. Progress Nuclear Energy 108, 295-309 (2018)

22. Dai, Q., Cao, Q., Chen, Y.: Frequency analysis of rotating truncated conical shells using the Haar wavelet method. Appl. Math. Model. 57, 603-613 (2018)

23. Singh, R., Garg, H., Guleria, V.: Haar wavelet collocation method for laneemden equations with dirichlet, neumann and neumannrobin boundary conditions. J. Comput. Appl. Math. 346, 150-161 (2019)

24. Islam, S., Sarler, B., Aziz, I., Haq, F.: Haar wavelet collocation method for the numerical solution of boundary layer fluid flow problems. Int. J. Therm. Sci. 50, 686-697 (2011) 
25. Akram, G., Rehman, H.U.: Numerical solution of eighth order boundary value problems in reproducing kernel space. Numer. Algorithms 62, 527-540 (2013). https://doi.org/10.1007/s11075-012-9608-4

26. Inc, M., Evans, D.J.: An efficient approach to approximate solutions of eighth-order boundary value problems. Int. J. Comput. Math. 81(6), 685-692 (2004)

27. Porshokouhi, M.G., Ghanbari, B., Gholami, M., Rashidi, M.: Numerical solution of eighth order boundary value problems with variational iteration method. Gen. Math. Notes 2(1), 128-133 (2011)

28. Siddiqi, S.S., Akram, G.: Solution of eighth-order boundary value problems using the nonpolynomial spline technique. Int. J. Comput. Math. 84(3), 347-368 (2007)

29. Mestrovic, M.: The modified decomposition method for eighth-order boundary value problems. Appl. Math. Comput. 188, 1437-1444 (2007)

30. He, J.H.: The variational iteration method for eighth-order initial-boundary value problems. Phys. Scr. 76, 680-682 (2007)

31. Noor, M.A., Mohyud-Din, S.T.: Variational iteration decomposition method for solving eighth-order boundary value problems. Differ. Equ. Nonlinear Mech. (2007). https://doi.org/10.1155/2007/19529

32. Golbabai, A., Javidi, M.: Application of homotopy perturbation method for solving eighth order boundary value problems. Appl. Math. Comput. 191, 334-346 (2007)

Publisher's Note Springer Nature remains neutral with regard to jurisdictional claims in published maps and institutional affiliations. 